\title{
Age is the Main Determinant of COVID-19 Related in-Hospital Mortality with Minimal Impact of Pre- Existing Comorbidities: A Retrospective Cohort Study.
}

M.T.H.M. Henkens ( $\square$ michiel.henkens@mumc.nl )

CARIM, Maastricht University Medical Center
A.G. Raafs
CARIM, Maastricht University Medical Center

J.A.J. Verdonschot

Maastricht University Medical Center

M. Linschoten

University Medical Center Utrecht, Utrecht University

M. van Smeden

University Medical Center Utrecht, Utrecht University

P. Wang

Maastricht University Medical Center

B.H.M. van der Hooft

Maastricht University Medical Center

\section{R. Tieleman}

Martini Hospital

\section{M.L.F. Janssen}

Maastricht University Medical Center

\section{R.M.A. ter Bekke}

Maastricht University Medical Center

\section{M.R. Hazebroek}

Maastricht University Medical Center

\section{I.C.C. van der Horst}

Maastricht University Medical Center

\section{F.W. Asselbergs}

University Medical Center Utrecht, Utrecht University

\section{F.J.H. Magdelijns}

Maastricht University Medical Center

\section{S.R.B. Heymans}

CARIM, Maastricht University Medical Center 
the CAPACITY-COVID collaborative consortium

University Medical Center Utrecht, Utrecht University

\section{Research Article}

Keywords: COVID-19, Mortality, Hospitalization, Netherlands, Mediation analysis

Posted Date: October 7th, 2021

DOl: https://doi.org/10.21203/rs.3.rs-955049/v1

License: (c) (1) This work is licensed under a Creative Commons Attribution 4.0 International License. Read Full License

Version of Record: A version of this preprint was published at BMC Geriatrics on March 5th, 2022. See the published version at https://doi.org/10.1186/s12877-021-02673-1. 


\section{Abstract}

Background: Age and comorbidities increase COVID-19 related in-hospital mortality risk, but the extent by which comorbidities mediate the impact of age remains unknown.

Methods: In this multicenter retrospective cohort study of 45 Dutch hospitals, 4,806 proven COVID-19 patients hospitalized in Dutch hospitals (between February and July 2020) from the CAPACITY-COVID registry were included (age 69 [58-77] years, 64\% men). The primary outcome was defined as a combination of in-hospital mortality or discharge with palliative care. Logistic regression analysis was performed to analyze the associations between sex, age, and comorbidities with in-hospital mortality. The effect of comorbidities on the relation of age with in-hospital mortality was evaluated using mediation analysis.

Results: In-hospital COVID-19 related mortality occurred in 1,108 (23\%) patients, 836 (76\%) were aged $\geq 70$ years $(70+)$. Both age $70+$ and female sex were univariably associated with outcome (odds ratio [OR]4.68, 95\%confidence interval [4.02-5.45], OR0.68[0.59-0.79], respectively; both $p<0.001)$. All comorbidities were univariably associated with outcome $(p<0.001)$, and all but dyslipidemia remained significant after adjustment for age70+ and sex. The impact of comorbidities was attenuated after agespline adjustment, only leaving female sex, diabetes mellitus (DM), chronic kidney disease (CKD), and chronic pulmonary obstructive disease (COPD) significantly associated (female OR0.65[0.55-0.75], DM OR1.47[1.26-1.72], CKD OR1.61[1.32-1.97], COPD OR1.30[1.07-1.59]). Pre-existing comorbidities in older patients negligibly $(<6 \%$ in all comorbidities) mediated the association between higher age and outcome.

Conclusions: Age is the main determinant of COVID-19 related in-hospital mortality, with negligible mediation effect of pre-existing comorbidities.

Trial registration: CAPACITY-COVID (NCT04325412).

\section{Background}

COVID-19 is a pandemic infectious disease caused by the severe acute respiratory syndrome coronavirus 2 (SARS-CoV-19), which already accounts for over 4.1 million deaths worldwide (1). COVID-19 related mortality is particularly high in the elderly (2-6). Consequently, in the Netherlands, $90 \%$ of the deceased patients is 70 years or older (7).

Pre-existing comorbidities such as hypertension, diabetes mellitus (DM), dyslipidemia, chronic kidney disease (CKD), chronic obstructive pulmonary disease (COPD), and history of cardiac diseases are also associated with an increased risk of COVID-19 related (in-hospital) mortality (8-12). However, these comorbidities are also known to be more prevalent in the elderly $(8,9,13)$. It remains unknown to which extent the higher in-hospital mortality rate is mediated by the higher prevalence of these comorbidities in the elderly. 
The aim of this study was to explore to which extent existing comorbidities mediate the increased risk of COVID-19 related in-hospital mortality relative to age in a registry of 4,806 Dutch COVID-19 patients.

\section{Methods}

\section{Study design and population}

CAPACITY-COVID (NCT04325412) is an international patient registry established to investigate the role of cardiovascular disease in the COVID-19 pandemic $(14,15)$. The details of this registry have been outlined in detail previously (14). In short, adult patients ( $\geq 18$ years) with (highly suspected) COVID-19 admitted to one of the participating hospitals were included in this registry (14). Forty-five Dutch hospitals contributed to the registry. For the current analysis, consecutive patients from all participating Dutch hospitals with proven COVID-19 infection based on at least one positive PCR for SARS-CoV-2 and/or a chest CT scan strongly suggestive for SARS-CoV-2 infection, hospitalized between January and July 2020 (first COVID-19 wave in the Netherlands), were included (16). In-hospital mortality was defined as a combination of in-hospital mortality or discharge with palliative care. The study was performed according to the Helsinki declaration and local ethics approval was obtained in all participating hospitals. Consent (when required by local ethical committees of all participating centers (METC, Medisch Ethische Toetsingscomissie) was obtained by either opt-in or opt-out approaches, following local regulations.

\section{Statistical analyses}

Variables are displayed as numbers (percentage), mean \pm standard deviation (SD) or median and inter quartile ranges [IQR], as appropriate. Normality was assessed visually using Q-Q plots. Comparisons between groups were performed using chi-square tests for categorical data and for continuous variables by independent sample T-test or Mann Whitney-U test depending on normality of the distribution. Unadjusted binary logistic regression analysis was performed to analyze the associations between sex, age 60+, age 70+, a reported medical history of comorbidities (hypertension, DM, dyslipidemia, CKD, COPD, and a medical history of cardiac disease) and the comorbidity count (a sum of the presence of the before mentioned comorbidities categorized as 0 co-morbidities, 1-2 co-morbidities, and $>2$ comorbidities) with in-hospital mortality. Definitions of pre-existing comorbidities as specified in the case report form are provided in the Supplemental Methods; Additional file 1). Subsequently, adjusted binary logistic regression analysis - with adjustment for age 70+ and sex - was performed to associate comorbidities and the comorbidity count with in-hospital mortality. Additionally, given the non-linear relationship between age and in-hospital mortality, age-restricted cubic spline adjusted logistic regression models were constructed for sex and the comorbidities(17).

$\mathrm{R}$ mediation package 4.5.0 was used to perform a mediation analysis (18). The mediation analysis tests whether a clinical variable (in this case age 70+) affects outcome through a mediator variable (in this case the co-morbidities) and to which extent. This divides the total effect of the model into a direct effect, called the average direct effect (ADE), and an indirect effect, called the average causal mediation effect (ACME). The proportion of ADE and ACME and the 95\% confidence intervals (using 1000x bootstrapping) 
were calculated and visualized for each comorbidity studied. Statistical analyses were conducted in R, and figures were made using the packages ggplot2, forest plot (19-21). Statistical significance was defined as a $P$ value $<0.05$.

\section{Results}

\section{Patient characteristics}

In total, 4,806 patients fulfilled the inclusion criteria and were included in this study. Clinical characteristics stratified by age $<70$ and $\geq 70$ years are outlined in Table 1 . The median age was 69 [5877] years, and approximately two-third were men $(63 \%, \mathrm{~N}=3,051)$. All pre-existing comorbidities were more prevalent in the elderly (aged $\geq 70$ years, all $p<0.001$ ). Male patients more often had dyslipidemia ( $46 \% \mathrm{vs}$ $39 \%$ ), a history of cardiac disease (39\% vs $30 \%$, especially coronary artery disease and arrhythmias) and presence of two or more comorbidities (39\% vs $33 \%$, Additional file 2 ). 
Table 1

Clinical characteristics of patients aged $<70$ and aged $\geq 70(70+)$ years.

\begin{tabular}{|c|c|c|c|c|}
\hline & $\begin{array}{l}<70 \\
(N=2,504)\end{array}$ & $\begin{array}{l}70+ \\
(N=2,302)\end{array}$ & $\begin{array}{l}\text { Total } \\
(\mathrm{N}=4,806)\end{array}$ & P-value \\
\hline \multicolumn{5}{|l|}{ Clinical Presentation } \\
\hline Age, years & 58 [51-64] & 77 [73-82] & 69 [58-77] & $<0.001$ \\
\hline Female & $910(36 \%)$ & $845(37 \%)$ & $1755(37 \%)$ & 0.793 \\
\hline $\mathrm{BMI}, \mathrm{kg} / \mathrm{m}^{2}$ & $27.9[25.1-31.2]$ & $26.7[24.0-30.1]$ & $27.3[24.5-30.8]$ & $<0.001$ \\
\hline Temperature, ${ }^{\circ} \mathrm{C}$ & $37.9 \pm 1.1$ & $37.7 \pm 1.1$ & $37.8 \pm 1.1$ & $<0.001$ \\
\hline Heart rate, bpm & 91 [80-103] & 86 [75-99] & 89 [77-101] & $<0.001$ \\
\hline Systolic BP, mmHg & $133 \pm 21$ & $136 \pm 24$ & $135 \pm 23$ & $<0.001$ \\
\hline Diastolic BP, mmHg & $78 \pm 14$ & $75 \pm 15$ & $77 \pm 15$ & $<0.001$ \\
\hline Breathing rate, rpm & $22[18-26]$ & $21[18-26]$ & 22 [18-26] & 0.620 \\
\hline Oxygen saturation, So2\% & 95 [92-97] & 95 [92-96] & 95 [92-97] & $<0.001$ \\
\hline \multicolumn{5}{|l|}{ Medical History, n (\%) } \\
\hline Hypertension & $1036(41 \%)$ & $1720(75 \%)$ & $2756(57 \%)$ & $<0.001$ \\
\hline Diabetes Mellitus & $512(20 \%)$ & $701(31 \%)$ & $1213(25 \%)$ & $<0.001$ \\
\hline Dyslipidemia & $764(31 \%)$ & $1303(57 \%)$ & 2067 (43\%) & $<0.001$ \\
\hline Chronic Kidney Disease & $127(5 \%)$ & $392(17 \%)$ & $519(11 \%)$ & $<0.001$ \\
\hline COPD & $206(8 \%)$ & $373(16 \%)$ & $579(12 \%)$ & $<0.001$ \\
\hline Cardiac disease & $488(20 \%)$ & $1218(53 \%)$ & $1706(36 \%)$ & $<0.001$ \\
\hline Arrhyth./Conduc. & $160(6 \%)$ & $612(27 \%)$ & $772(16 \%)$ & $<0.001$ \\
\hline Heart Failure & $46(2 \%)$ & $228(10 \%)$ & $274(6 \%)$ & $<0.001$ \\
\hline Coronary Artery Disease & $249(10 \%)$ & $574(25 \%)$ & $823(17 \%)$ & $<0.001$ \\
\hline Valvular Heart Disease & $50(2 \%)$ & $201(9 \%)$ & $251(5 \%)$ & $<0.001$ \\
\hline Comorbidity count & & & & $<0.001$ \\
\hline 0 comorbidities & $1073(43 \%)$ & $250(11 \%)$ & $1323(28 \%)$ & \\
\hline 1-2 comorbidities & $896(36 \%)$ & $842(37 \%)$ & $1738(36 \%)$ & \\
\hline
\end{tabular}

Arrhyth = arrhythmias; $\mathrm{BMI}=$ body mass index; $\mathrm{BP}=$ blood pressure; Conduc = conduction disorders; COPD = chronic obstructive pulmonary disease. 


\begin{tabular}{|c|c|c|c|c|}
\hline & $\begin{array}{l}<70 \\
(N=2,504)\end{array}$ & $\begin{array}{l}70+ \\
(N=2,302)\end{array}$ & $\begin{array}{l}\text { Total } \\
(\mathrm{N}=4,806)\end{array}$ & P-value \\
\hline$>2$ comorbidities & $535(21 \%)$ & 1210 (53\%) & 1745 (36\%) & \\
\hline
\end{tabular}

Median duration of hospitalization stay till the study endpoint was 7 [4 - 15] days. In-hospital mortality $(\mathrm{N}=1,066)$ or palliative discharge $(\mathrm{N}=42)$ occurred in $1,108(23 \%)$ patients, of which $836(76 \%)$ were aged $70+$ and $272(24 \%)$ were aged $<70(p<0.001 ;$ Table 2$)$. The observed and predicted association between age (splined adjusted) and in-hospital mortality are shown in Figure 1. Male sex and the individual comorbidities were more prevalent, and comorbidity count per patient was higher in the in-hospital mortality group ( $<<0.001$, Table 2$)$. In total, $1,312(27 \%)$ patients were referred to the intensive care unit (ICU) during hospitalization, of which 446 were aged $70+(34 \%)$ and $866(66 \%)$ were aged $<70(p<0.001)$. 
Table 2

Clinical characteristics of patients with and without event.

$\begin{array}{llll}\begin{array}{l}\text { No mortality or Palliative } \\ \text { Care }\end{array} & \begin{array}{l}\text { Mortality or Palliative } \\ \text { Care }\end{array} & \begin{array}{l}\text { Total } \\ (N=4,806)\end{array} & \begin{array}{l}\text { P- } \\ \text { value }\end{array} \\ (\mathrm{N}=3,698) & (\mathrm{N}=1,108) & & \end{array}$

Clinical Presentation

\begin{tabular}{|c|c|c|c|c|}
\hline Age, years & 65 [56-74] & 76 [70-82] & 69 [58-77] & $<0.001$ \\
\hline Female & $1423(39 \%)$ & $332(30 \%)$ & $1755(37 \%)$ & $<0.001$ \\
\hline $\mathrm{BMI}, \mathrm{kgm}^{-2}$ & 27.5 [24.7-30.8] & $26.8[24.1-30.5]$ & $\begin{array}{l}27.3[24.5- \\
30.8]\end{array}$ & 0.010 \\
\hline Temperature, ${ }^{\circ} \mathrm{C}$ & $37.8 \pm 1.1$ & $37.8 \pm 1.1$ & $37.8 \pm 1.1$ & 0.454 \\
\hline Heart rate, bpm & 89 [78-100] & 90 [77-103] & 89 [77-101] & 0.087 \\
\hline systolic BP, mmHg & $135 \pm 22$ & $134 \pm 25$ & $135 \pm 23$ & 0.301 \\
\hline diastolic BP, mmHg & $77 \pm 14$ & $73 \pm 16$ & $77 \pm 15$ & $<0.001$ \\
\hline Breathing rate, rpm & 20 [17-25] & $24[19-28]$ & $22[18-26]$ & $<0.001$ \\
\hline $\begin{array}{l}\text { Oxygen saturation, } \\
\text { So } 2 \%\end{array}$ & 95 [93-97] & 94 [91-96] & 95 [92-97] & $<0.001$ \\
\hline \multicolumn{5}{|l|}{ Medical History } \\
\hline Hypertension & 1979 (54\%) & 777 (70\%) & $2756(57 \%)$ & $<0.001$ \\
\hline Diabetes Mellitus & $835(23 \%)$ & $378(34 \%)$ & $1213(25 \%)$ & $<0.001$ \\
\hline Dyslipidemia & $1479(40 \%)$ & $588(53 \%)$ & 2067 (43\%) & $<0.001$ \\
\hline $\begin{array}{l}\text { Chronic Kidney } \\
\text { Disease }\end{array}$ & $311(8 \%)$ & 208 (19\%) & $519(11 \%)$ & $<0.001$ \\
\hline COPD & $389(11 \%)$ & $190(17 \%)$ & $579(12 \%)$ & $<0.001$ \\
\hline Cardiac disease & $1170(32 \%)$ & $536(48 \%)$ & $1706(36 \%)$ & $<0.001$ \\
\hline Arrhyth./Conduc. & $509(14 \%)$ & $263(24 \%)$ & $772(16 \%)$ & $<0.001$ \\
\hline Heart Failure & $168(5 \%)$ & $106(10 \%)$ & $274(6 \%)$ & $<0.001$ \\
\hline $\begin{array}{l}\text { Coronary Artery } \\
\text { Disease }\end{array}$ & $540(15 \%)$ & $283(26 \%)$ & $823(17 \%)$ & $<0.001$ \\
\hline $\begin{array}{l}\text { Valvular Heart } \\
\text { Disease }\end{array}$ & $155(4 \%)$ & $96(9 \%)$ & $251(5 \%)$ & $<0.001$ \\
\hline
\end{tabular}

Arrhyth = arrhythmias; $\mathrm{BMI}=$ body mass index $\mathrm{BP}=$ blood pressure; Conduc = conduction disorders; COPD = chronic obstructive pulmonary disease. 


\begin{tabular}{|lllll|}
\hline & $\begin{array}{l}\text { No mortality or Palliative } \\
\text { Care } \\
(\mathbf{N}=3,698)\end{array}$ & $\begin{array}{l}\text { Mortality or Palliative } \\
\text { Care } \\
(\mathbf{N}=1,108)\end{array}$ & $\begin{array}{l}\text { Total } \\
(\mathbf{N}=4,806)\end{array}$ & $\begin{array}{l}\text { P- } \\
\text { value }\end{array}$ \\
\hline Comorbidity count & & & $1323(28 \%)$ \\
\hline 0 comorbidities & $1164(32 \%)$ & $159(14 \%)$ & $1738(36 \%)$ \\
\hline $1-2$ comorbidities & $1354(37 \%)$ & $584(35 \%)$ & $1745(36 \%)$ \\
\hline$>2$ comorbidities & $1180(32 \%)$ & $565(51 \%)$ & \\
\hline $\begin{array}{l}\text { Arrhyth = arrhythmias; BMI = body mass index; BP = blood pressure; Conduc = conduction disorders; } \\
\text { COPD = chronic obstructive pulmonary disease. }\end{array}$
\end{tabular}

\section{Univariable and multivariable associations and mediation effect of comorbidities and in-hospital mortality}

Higher age (70+), male sex, and the individual comorbidities were all significantly associated with inhospital mortality in univariable analysis (all $p<0.001$; Figure $2 A$ ). Hypertension, DM, CKD, COPD and a history of cardiac disease remained significantly associated after adjustment for age 70+ and sex (hypertension odds ratio [OR] 1.29, 95\% confidence interval [CI] 1.10-1.51, $\mathrm{p}=0.001$; DM OR 1.55 [1.331.81], $p<0.001$; dyslipidemia OR 1.15 [1.00-1.34], $p=0.053$; CKD OR 1.74 [1.43-2.13], $p<0.001$; COPD OR 1.41 [1.15-1.72], $p<0.001$; history of cardiac disease OR 1.22 [1.05-1.41], $p=0.010$, Figure 2B). Additionally, given the non-linear relationship between age and in-hospital mortality, age-spline adjusted analysis was performed for the association of sex and all comorbidities with in-hospital mortality (Figure 2C). The effects were attenuated after age-spline adjustment, only leaving sex, DM, CKD, and COPD significantly associated with in-hospital mortality (female OR 0.65 [0.55-0.75], p<0.001; DM OR 1.47 [1.26-1.72], $p<0.001$; CKD OR 1.61 [1.32-1.97], $p<0.001$; COPD OR 1.30 [1.07-1.59], $p=0.010$, Figure 2C).

Uni- and multivariable (spline-adjusted) analysis of comorbidity count revealed a significant, univariable association with in-hospital mortality ( $p<0.001$; Additional file 3 ), which remained significant in the multivariable model with an attenuated effect. In the age-spline multivariable adjusted analysis, the association of the comorbidity count was not significant for a comorbidity count of 1-2 (OR 1.15 [0.9-1.4]) and attenuated for $>2$ comorbidity count (OR 1.4 [1.1-1.7]).

All individual comorbidities significantly mediated the association between age 70+ and in-hospital mortality (ACME; Figure 3). However, the proportion of this effect was below $6 \%$ for all comorbidities, and thereby the increased risk of in-hospital mortality in the elderly was explained mainly by the direct effect (ADE; Figure 3 ) of age 70+ within this analysis (Figure 3). Additionally, a mediation analysis was performed to quantify the mediation effect of a comorbidity count $>2$ on the association between age and in-hospital mortality. Although the proportion of this effect was higher than the comorbidities separately, it remained minimal ( $8 \%, p<0.001$, Additional file 4). 
Age-spline plots showed a steep increase in the predicted risk of in-hospital mortality from 60 years and higher (Figure 1; stratified by sex and the individual comorbidities is shown in Additional file 5).

Correspondingly, in-hospital mortality rates were significantly lower in patients $<60$ years when compared to the older patients (age<60 years, $6 \%(N=87)$ vs $30 \%(N=1,021), p<0.001$; Additional file 6). All comorbidities also occurred less frequently present in this younger patient group (Additional file 6). None of the co-morbidities, except for history of cardiac disease, were significantly associated with in-hospital mortality in univariable analysis in patients $<60$ years (hypertension OR 1.31 [0.83-2.06], $p=0.240$; DM OR 1.38 [0.82-2.35], $p=0.228$; dyslipidemia OR 1.26 [0.76-2.10], $p=0.367$; CKD OR 2.33 [0.96-5.66], $p=0.062$; COPD OR 0.62 [0.19-2.00], $p=0.421$; history of cardiac disease OR 1.90 [1.10-3.27], $p=0.021$, Additional file 7). In the total patient population, age $60+$ was significantly associated with in-hospital mortality (OR 6.35[5.05-7.98], $p<0.001$; Additional file 7A).

\section{Discussion}

This study reveals that higher age is the main determinant of COVID-19 related in-hospital mortality in the Netherlands. While pre-existing comorbidities (including hypertension, DM, dyslipidemia, CKD, COPD, and cardiac diseases) are more prevalent in the elderly, their contributing effect to COVID-19 related inhospital mortality is minimal.

Over the last months, studies revealed multiple risk factors independently associated with COVID-19 related (in-hospital) mortality, including higher age, pre-existing co-morbidities, and male sex, which is in line with current findings $(9,10,22-25)$. A recent study of 2,273 COVID-19 hospitalized Dutch patients showed that a mortality prediction model using ten clinical features including age, number of home medications, admission blood values urea nitrogen/LDH/albumin, oxygen saturation, blood gas $\mathrm{pH}$ and history of chronic cardiac disease, improved discrimination over age-based decision rules only (26). Nonetheless, no data regarding to which extent age and cardiovascular comorbidities contributed to the mortality risks were provided. We show that the contributing effect of pre-existing comorbidities was negligible relative to age.

In the Netherlands, the vast majority (90\%) of deceased patients due to COVID-19 is over 70 years of age. The prevalence of comorbidities among hospitalized COVID-19 patients is particularly high, especially in the elderly $(7,8)$. The current study included 4,806 patients from 45 Dutch - both academic and local hospitals, accounting for around $40 \%$ of all COVID-19 related hospitalizations between January 2020 and July 2020 in the Netherlands (7). Therefore, this population adequately represents the total hospitalized population during the first COVID-19 pandemic wave in the Netherlands. The higher prevalence of male COVID patients observed in the current registry and the increased mortality risk of males is in line with previous findings and likely due to a less effective viral control of the immune system in males $(24,27)$, together with the higher comorbidity rates in males compared to females $(24,28)$. The observed association of history of cardiac disease and in-hospital mortality in the young has been studied and discussed before by current consortium, which is mainly driven by the presence of coronary artery disease and severe (NYHA $\geq 3)$ heart failure (29). 
It is important to notice that during the first wave of COVID-19, the median age of people referred to the ICU department decreased when the total number of patients referred to the ICU department increased (30). Younger $(<70+)$ patients were more likely to be referred to the ICU department due to bed shortages. The lower percentage of mortality $(75 \%)$ of patients $70+$ in the current study is lower than the overall mortality rate in this age group in the Netherlands in 2020 (90\%) (31). This likely results from fewer referrals of elderly patients to the hospitals (possibly even independent of the presence of comorbidities) due to the overwhelming stress on hospitals during the first COVID-19 wave. However, even in the group aged $<60$ years - in whom likely no or limited referral "restrictions" were present - the effect of pre-existing comorbidities on mortality was limited in the current study, indicating that age as such is likely the main driver of in-hospital mortality.

Our study revealed that pre-existing co-morbidities, highly prevalent in the elderly, contributed minimally to the larger in-hospital mortality rates when compared to age. Moreover, the contribution of the comorbidities to outcome in patients aged $<60$ years was limited to a history of cardiac disease without any significant association for DM, CKD, and COPD. As a result, the present study stresses the need for primary preventive efforts to protect the elderly (males) from an infection with SARS-CoV-19.

\section{Limitations}

Data concerning the patients' frailty and the reasons for not referring patients to the hospital or the ICU, were not collected within this registry. To which extent the lower hospital and ICU referral rate of the elderly and frailty contributed to the in-hospital mortality rate could therefore not be assessed and requires further research. Such research could help to understand better the association of higher age and in-hospital mortality beyond the comorbidities and might guide decision making on treatment, counselling and admission to high care facilities in the future.

\section{Conclusion}

Age is the main determinant of COVID-19 related in-hospital mortality, which is negligibly mediated by pre-existing comorbidities in the Netherlands.

\section{Abbreviations}

Average direct effect $=\mathrm{ADE}$

Average causal mediation effect $=\mathrm{ACME}$

Chronic kidney disease $=$ CKD

Chronic obstructive pulmonary disease $=$ COPD

Coronavirus 2 = SARS-CoV-19

Page $11 / 21$ 
Diabetes mellitus $=\mathrm{DM}$

Intensive care unit $=\mathrm{ICU}$

\section{Declarations}

\section{Ethics approval and consent to participate}

The study was performed according to the Helsinki declaration and local ethics approval was obtained in all participating hospitals. Consent (when required by local ethical committees) was obtained by either opt-in or opt-out approaches, following local regulations.

\section{Consent for publication}

Not applicable.

\section{Availability of data and materials}

The datasets used and/or analyzed during the current study are available from the corresponding author on reasonable request.

\section{Competing interests}

The authors declare that they have no competing interests.

\section{Funding}

CAPACITY-COVID is supported by the Dutch Heart Foundation (2020B006 CAPACITY), Novartis Global, Novo Nordisk Nederland, Servier Nederland and Daiichi Sankyo Nederland. Marijke Linschoten is supported by the Alexandre Suerman Stipend of the University Medical Center Utrecht. Folkert W. Asselbergs is supported by CardioVasculair Onderzoek Nederland 2015-12 eDETECT and by the NIHR University College London Hospitals Biomedical Research Centre.

\section{Author's contributions}

$\mathrm{MH}, \mathrm{AR}$ and $\mathrm{ML}$ were in the lead for data collection. $\mathrm{MH}, \mathrm{AR}$ and $\mathrm{JV}$ were in the lead for the conception and design of the study, the analysis and interpretation of the data. MvS and PW helped with the analysis and interpretation of data. MH and AR were also in the lead for drafting the article. JV, ML, MvS, BvH, RT, MJ, RtB, MHazebroek, IvdH, FA, FM and SH provided additive contribution to the conception, design and 
interpretation of the data and critically revised the draft. All authors fulfil all three of the ICMJE guidelines for authorship: 1) substantial contributions to conception and design, acquisition of data, or analysis and interpretation of data; 2) drafting the article or revising it critically for important intellectual content; and 3) final approval of the version to be published.

\section{Acknowledgements}

We want to express our gratitude and appreciation to all participating sites and researchers' part of the CAPACITY-COVID collaborative consortium and all (research) professionals that have contributed to the data collection. CAPACITY-COVID gratefully acknowledges the following organizations for their assistance in the development of the registry and/or coordination regarding the data registration in the collaborating centers: partners of the Dutch CardioVascular Alliance (DCVA), the Dutch Association of Medical Specialists (FMS) and the British Heart Foundation Centers of Research Excellence. In addition, the consortium is thankful for the endorsement of the CAPACITY-COVID initiative by the European Society of Cardiology (ESC), the European Heart Network (EHN) and the Society for Cardiovascular Magnetic Resonance (SCMR). Furthermore, the consortium appreciates the endorsement of CAPACITY-COVD as a flagship research project within the National Institute for Health Research (NIHR)/British Heart Foundation (BHF) Partnership framework for COVID-19 research.

\section{CAPACITY-COVID collaborative consortium}

Al-Windy, NYY ${ }^{13}$; Anthonio, RL ${ }^{14}$; Berg ten, $\mathrm{JM}^{15}$; Dorman, $\mathrm{HGR}^{16}$; Drost, $\mathrm{JT}^{17}$; Emans, $\mathrm{ME}^{18}$; Gilst van, $\mathrm{WH}^{19}$; Groenemeijer, $\mathrm{BE}^{20}$; Haerkens-Arends, $\mathrm{HE}^{21}$; Harst van der, $\mathrm{P}^{4}$; Heijden van der, $\mathrm{DJ}^{22}$; Hermanides, $\mathrm{RS}^{23}$; Hermans-van Ast, W24; Hessen van, MWJ ${ }^{25}$; Jewbali, LS ${ }^{26,27}$; Kesteren van, HAM $^{28}$; Kietselaer, $\mathrm{BLJH}{ }^{29}$; Koning, $\mathrm{AMH}^{30}$; Kuijper, $\mathrm{AFM}^{31}$; Linden van der, $\mathrm{MMJM}^{32}$; Linssen, $\mathrm{GCM}^{33}$; Martens, $\mathrm{FMAC}^{34}$; Meer van der, $\mathrm{P}^{35}$; Meijs, MFL ${ }^{36}$; Monraats, $\mathrm{PS}^{37}$; Mosterd, $\mathrm{A}^{38}$; Nierop, $\mathrm{PR}^{39}$; Ofwegen van-Hanekamp, $\mathrm{CEE}^{40}$; Pinto, $\mathrm{YM}^{41,42}$; Pisters, $\mathrm{R}^{43}$; Schaap, ${ }^{44,45}$; Schellings, DAAM ${ }^{46}$; Siebelink, $\mathrm{HJ}^{47}$; Smits, $\mathrm{PC}^{48}$; Tieleman, RG7; Tio, RA ${ }^{49,50}$; Tjong, FVY ${ }^{41,42,51}$; Uil den, $\mathrm{CA}^{26,27}$; Verschure, $\mathrm{DO}^{52}$; Vries de, JK ${ }^{53}$; Wal van de, $\mathrm{RMA}^{54}$; Watering van de, DJ ${ }^{55}$; Westendorp, ICD ${ }^{56}$; Westendorp, $\mathrm{PHM}^{57}$; Wierda, $\mathrm{E}^{58}$; Woudstra, $\mathrm{P}^{59}$; Wu, $\mathrm{KW}^{60} ;$ Zee van der, $\mathrm{PM}^{61}$.

${ }^{1}$ Department of Cardiology, CARIM, Maastricht University Medical Center, Maastricht, the Netherlands.

${ }^{2}$ Netherlands Heart Institute (NLHI), Utrecht, The Netherlands.

${ }^{3}$ Department of Clinical Genetics, CARIM, Maastricht University Medical Center, Maastricht, the Netherlands.

${ }^{4}$ Department of Cardiology, Division of Heart and Lungs, University Medical Center Utrecht, Utrecht University, Utrecht, the Netherlands. 
${ }^{5}$ UMCU-Julius Center for Health Sciences and Primary Care, University Medical Center Utrecht, Utrecht University, Utrecht, the Netherlands.

${ }^{6}$ Department of Internal Medicine, Maastricht University Medical Center, Maastricht, the Netherlands.

${ }^{7}$ Department of Cardiology, Martini Hospital, Groningen, The Netherlands.

${ }^{8}$ Department of Clinical Neurophysiology, Maastricht University Medical Center, Maastricht, the Netherlands.

${ }^{9}$ Department of Intensive Care, Maastricht University Medical Center, Maastricht, the Netherlands.

10 Institute of Cardiovascular Science, Faculty of Population Health Sciences, University College London, London, UK.

${ }^{11}$ Health Data Research UK and Institute of Health Informatics, University College London, London, UK.

12 Department of Cardiovascular Research, University of Leuven, Leuven, Belgium.

13 Department of Cardiology, Gelre Hospital Zutphen, Zutphen, the Netherlands

14 Department of Cardiology, Treant Zorggroep, Emmen, the Netherlands

15 Department of Cardiology, St. Antonius Hospital, Nieuwegein, the Netherlands

16 Department of Cardiology, Bravis Hospital, Roosendaal, the Netherlands

17 Department of Cardiology, Saxenburgh Medical Center, Hardenberg, the Netherlands

18 Department of Cardiology, Ikazia Hospital, Rotterdam, the Netherlands

${ }^{19}$ Department of Cardiology, University Medical Center Groningen, Groningen, the Netherlands

20 Department of Cardiology, Gelre Hospital Apeldoorn, Apeldoorn, the Netherlands

21 Department of Cardiology, Jeroen Bosch Hospital, 's-Hertogenbosch, the Netherlands

22 Department of Cardiology, Haaglanden Medical Center, the Hague, the Netherlands

23 Department of Cardiology, Isala Hospital, Zwolle, the Netherlands

24 Durrer Center, Netherlands Heart Institute, Utrecht, the Netherlands

25 Department of Cardiology, Groene Hart Hospital, Gouda, the Netherlands

${ }^{26}$ Department of Cardiology, Erasmus MC University Medical Center, Rotterdam, the Netherlands

27 Department of Intensive Care, Erasmus MC University Medical Center, Rotterdam, the Netherlands 
Department of Cardiology, Admiraal de Ruyter Hospital, Goes, the Netherlands

${ }^{29}$ Department of Cardiology, Zuyderland Medical Center, Heerlen, the Netherlands

30 Department of Gynecology, Amstelland Hospital, Amstelveen, the Netherlands

31 Department of Cardiology, Spaarne Gasthuis, Haarlem, the Netherlands

32 Department of Cardiology, Franciscus Vlietland, Schiedam, the Netherlands

33 Department of Cardiology, Hospital Group Twente, Almelo, the Netherlands

34 Department of Cardiology, Deventer Hospital, Deventer, the Netherlands

35 Department of Cardiology, LangeLand Hospital, Zoetermeer, the Netherlands

${ }^{36}$ Department of Cardiology, Thorax Center Twente, Medisch Spectrum Twente, Enschede, the Netherlands

37 Department of Cardiology, Elizabeth-TweeSteden Hospital, Tilburg, the Netherlands

38 Department of Cardiology, Meander Medical Center, Amersfoort, the Netherlands

${ }^{39}$ Department of Cardiology, Franciscus Gasthuis, Rotterdam, the Netherlands

40 Department of Cardiology, Diakonessenhuis, Utrecht, the Netherlands

${ }^{41}$ Heart Center, Amsterdam University Medical Center, University of Amsterdam, the Netherlands

42 Department of Clinical and Experimental Cardiology, Amsterdam Cardiovascular Sciences, Amsterdam, the Netherlands

43 Department of Cardiology, Rijnstate Hospital, Arnhem, the Netherlands

44 Department of Cardiology, Amphia Hospital, the Netherlands

45 The Dutch Network for Cardiovascular Research (WCN), Utrecht, the Netherlands

46 Department of Cardiology, Slingeland Hospital Doetinchem, the Netherlands

47 Department of Cardiology, Leiden University Medical Center, Leiden, the Netherlands

48 Department of Cardiology, Maasstad Hospital, Rotterdam, the Netherlands

49 Department of Cardiology, Catharina Hospital, Eindhoven, the Netherlands 
${ }^{50}$ Department of Educational Development and Research in the Faculty of Health, Medicine and Life Sciences, Catharina Hospital, Eindhoven, the Netherlands

${ }^{51}$ Department of Cardiology, Vrije Universiteit Amsterdam, Amsterdam Cardiovascular Sciences, Amsterdam, the Netherlands

${ }^{52}$ Department of Cardiology, Zaans Medical Center, Zaandam, the Netherlands

${ }^{53}$ Department of Internal Medicine, Antonius Hospital, Sneek, the Netherlands

54 Department of Cardiology, Bernhoven Hospital, Uden, the Netherlands

${ }^{55}$ Department of Cardiology, Albert Schweitzer Hospital, Dordrecht, the Netherlands

${ }^{56}$ Department of Cardiology, Rode Kruis Hospital, Beverwijk, the Netherlands

57 Department of Cardiology, Beatrix Hospital, Gorinchem, the Netherlands

${ }^{58}$ Department of Cardiology, Dijklander Hospital, Hoorn, the Netherlands

${ }^{59}$ Department of Cardiology, Medical Center Leeuwarden (MCL), Leeuwarden, the Netherlands

${ }^{60}$ Department of Cardiology, van Weel-Bethesda Hospital, Dirksland, the Netherlands

${ }^{61}$ Department of Cardiology, St. Jansdal Hospital, Harderwijk, the Netherlands

\section{References}

1. WHO COVID-19 Dashboard. Geneva: World Health Organization, 2020. Available online: https://covid19.who.int/ (last cited: 16-07-2021).

2. Sun P, Lu X, Xu C, Sun W, Pan B. Understanding of COVID-19 based on current evidence. J Med Virol. 2020;92(6):548-51.

3. Wu C, Chen X, Cai Y, Xia Ja, Zhou X, Xu S, et al. Risk Factors Associated With Acute Respiratory Distress Syndrome and Death in Patients With Coronavirus Disease 2019 Pneumonia in Wuhan, China. JAMA Intern Med. 2020;180(7):934-43.

4. Grasselli G, Greco M, Zanella A, Albano G, Antonelli M, Bellani G, et al. Risk Factors Associated With Mortality Among Patients With COVID-19 in Intensive Care Units in Lombardy, Italy. JAMA Intern Med. 2020;180(10):1345-55.

5. NHS Digital. Coronavirus (COVID-19): Shielded patients list 2020.

6. Zhou F, Yu T, Du R, Fan G, Liu Y, Liu Z, et al. Clinical course and risk factors for mortality of adult inpatients with COVID-19 in Wuhan, China: a retrospective cohort study. The Lancet. 2020;395(10229):1054-62. 
7. Rijksinstituut voor volksgezondheid (RIVM): Mortality rates, Coronadashboard, https://coronadashboard.rijksoverheid.nl/landelijk/sterfte (last cited: 16-7-2021). 2021.

8. Ariës MJH, van den Bergh JP, Beudel M, Boersma W, Dormans T, Douma RA, et al. [Clinical course of COVID-19 in the Netherlands: an overview of 2607 patients in hospital during the first wave]. Ned Tijdschr Geneeskd. 2021;165.

9. Posso M, Comas M, Román M, Domingo L, Louro J, González C, et al. Comorbidities and Mortality in Patients With COVID-19 Aged 60 Years and Older in a University Hospital in Spain. Arch Bronconeumol. 2020;56(11):756-8.

10. Zuccaro V, Celsa C, Sambo M, Battaglia S, Sacchi P, Biscarini S, et al. Competing-risk analysis of coronavirus disease 2019 in-hospital mortality in a Northern Italian centre from SMAtteo COvid19 REgistry (SMACORE). Sci Rep. 2021;11(1):1137.

11. Docherty AB, Harrison EM, Green CA, Hardwick HE, Pius R, Norman L, et al. Features of 20133 UK patients in hospital with covid-19 using the ISARIC WHO Clinical Characterisation Protocol: prospective observational cohort study. Bmj. 2020;369:m1985.

12. Williamson EJ, Walker AJ, Bhaskaran $\mathrm{K}$, Bacon S, Bates $\mathrm{C}$, Morton $\mathrm{CE}$, et al. Factors associated with COVID-19-related death using OpenSAFELY. Nature. 2020;584(7821):430-6.

13. Atkins JL, Masoli JAH, Delgado J, Pilling LC, Kuo C-L, Kuchel GA, et al. Preexisting Comorbidities Predicting COVID-19 and Mortality in the UK Biobank Community Cohort. The Journals of Gerontology: Series A. 2020;75(11):2224-30.

14. Linschoten M, Asselbergs FW. CAPACITY-COVID: a European Registry to determine the role of cardiovascular disease in the COVID-19 pandemic. Eur Heart J. 2020;41(19):1795-6.

15. consortium C-Cc, Group LS, Linschoten M, Uijl A, Schut A, Jakob C, et al. Clinical presentation, disease course and outcome of COVID-19 in hospitalized patients with and without pre-existing cardiac disease - a cohort study across eighteen countries. medRxiv. 2021:2021.03.11.21253106.

16. Wang Y, Kang H, Liu X, Tong Z. Combination of RT-qPCR testing and clinical features for diagnosis of COVID-19 facilitates management of SARS-CoV-2 outbreak. J Med Virol. 2020;92(6):538-9.

17. Frank E Harrell Jr (2021). rms: Regression Modeling Strategies. R package version 6.2-0. https://CRAN.R-project.org/package=rms.

18. Tingley D, Yamamoto T, Hirose K, Keele L, Imai K. Mediation: R package for causal mediation analysis. Journal of Statistical Software. 2014;59(5):1-38.

19. R Core Team (2021). R: A language and environment for statistical computing. R Foundation for Statistical Computing, Vienna, Austria. https://www.R-project.org/.

20. Max Gordon and Thomas Lumley (2020). forestplot: Advanced Forest Plot Using 'grid' Graphics. R package version 1.10.1. https://CRAN.R-project.org/package=forestplot.

21. H. Wickham. ggplot2: Elegant Graphics for Data Analysis. Springer-Verlag New York, 2016.

22. Zheng Z, Peng F, Xu B, Zhao J, Liu H, Peng J, et al. Risk factors of critical \& mortal COVID-19 cases: A systematic literature review and meta-analysis. J Infect. 2020;81(2):e16-e25. 
23. Mendes A, Serratrice C, Herrmann FR, Genton L, Périvier S, Scheffler M, et al. Predictors of In-Hospital Mortality in Older Patients With COVID-19: The COVIDAge Study. Journal of the American Medical Directors Association. 2020;21(11):1546-54.e3.

24. Gebhard C, Regitz-Zagrosek V, Neuhauser HK, Morgan R, Klein SL. Impact of sex and gender on COVID-19 outcomes in Europe. Biol Sex Differ. 2020;11(1):29.

25. Mohamed MO, Gale CP, Kontopantelis E, Doran T, de Belder M, Asaria M, et al. Sex Differences in Mortality Rates and Underlying Conditions for COVID-19 Deaths in England and Wales. Mayo Clin Proc. 2020;95(10):2110-24.

26. Ottenhoff MC, Ramos LA, Potters W, Janssen MLF, Hubers D, Hu S, et al. Predicting mortality of individual patients with COVID-19: a multicentre Dutch cohort. BMJ Open. 2021;11(7):e047347.

27. Peckham H, de Gruijter NM, Raine C, Radziszewska A, Ciurtin C, Wedderburn LR, et al. Male sex identified by global COVID-19 meta-analysis as a risk factor for death and ITU admission. Nat Commun. 2020;11(1):6317.

28. Schiffer VMMM, Janssen EBNJ, van Bussel BCT, Jorissen LLM, Tas J, Sels J-WEM, et al. The "sex gap" in COVID-19 trials: a scoping review. EClinicalMedicine. 2020:100652.

29. Linschoten M, Peters S, van Smeden M, Jewbali LS, Schaap J, Siebelink HM, et al. Cardiac complications in patients hospitalised with COVID-19. Eur Heart J Acute Cardiovasc Care. 2020;9(8):817-23.

30. Linschoten M, Nab L, van der Horst ICC, Tieleman R, Asselbergs FW. Response to "Early hydroxychloroquine but not chloroquine use reduces ICU admission in COVID-19 patients". Int J Infect Dis. 2021;103:560-1.

31. Rijksinstituut voor volksgezondheid (RIVM): Mortality rates, Coronadashboard. https://coronadashboardrijksoverheidnl/landelijk/sterfte. 2021.

\section{Figures}




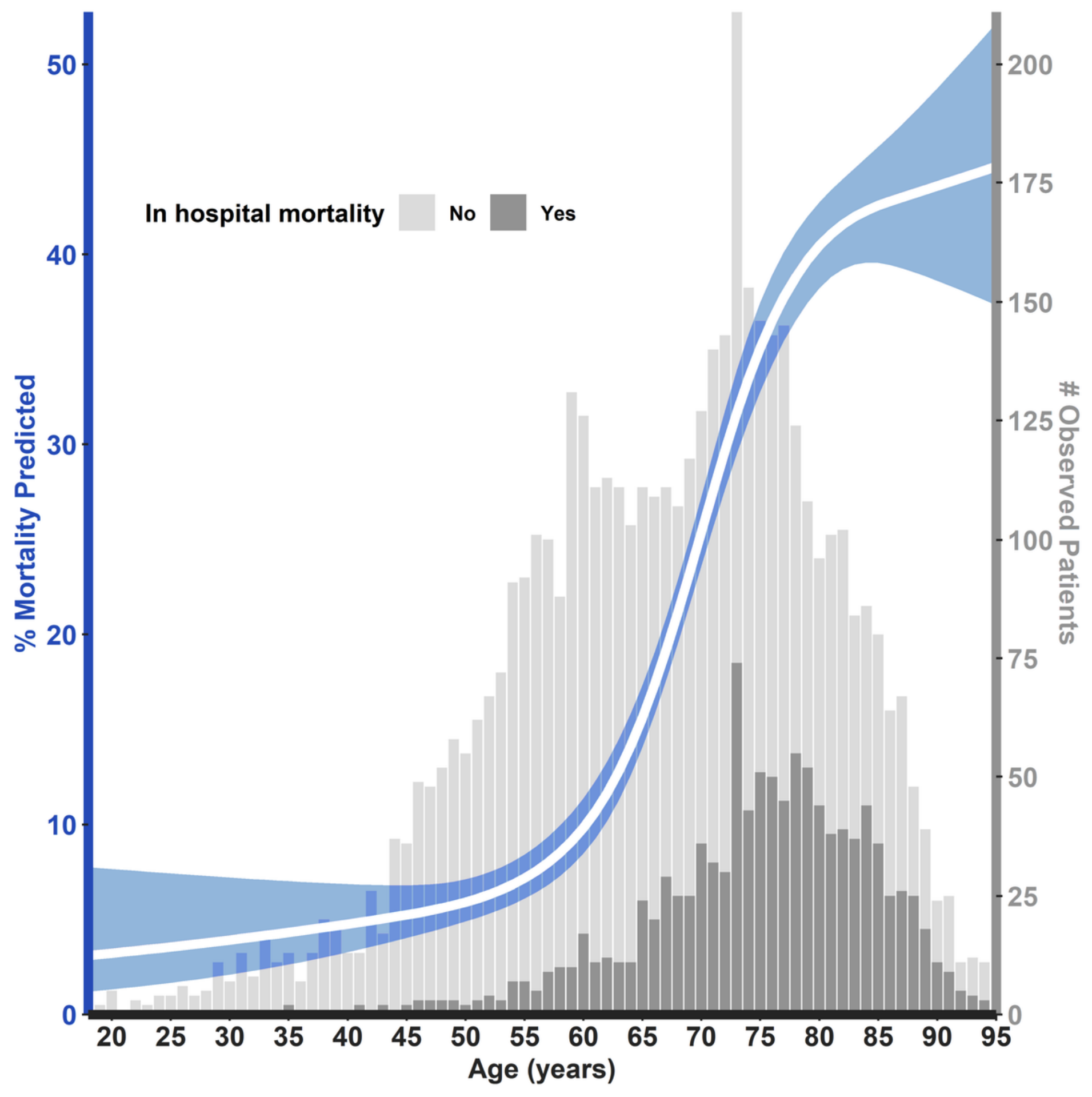

\section{Figure 1}

Predicted and observed in-hospital mortality (univariable age-restricted cubic spline adjusted). \% Mortality predicted: The white line represents the predicted mortality rates at different ages, accompanied by $95 \%$ confidence intervals in blue. \# Observed patients: The grey bars represent the number of patients observed within the different age categories. The light grey bars are the patients that were discharged without palliative care. The dark grey bars are the patients in which in-hospital mortality (or palliative discharge) occurred. 


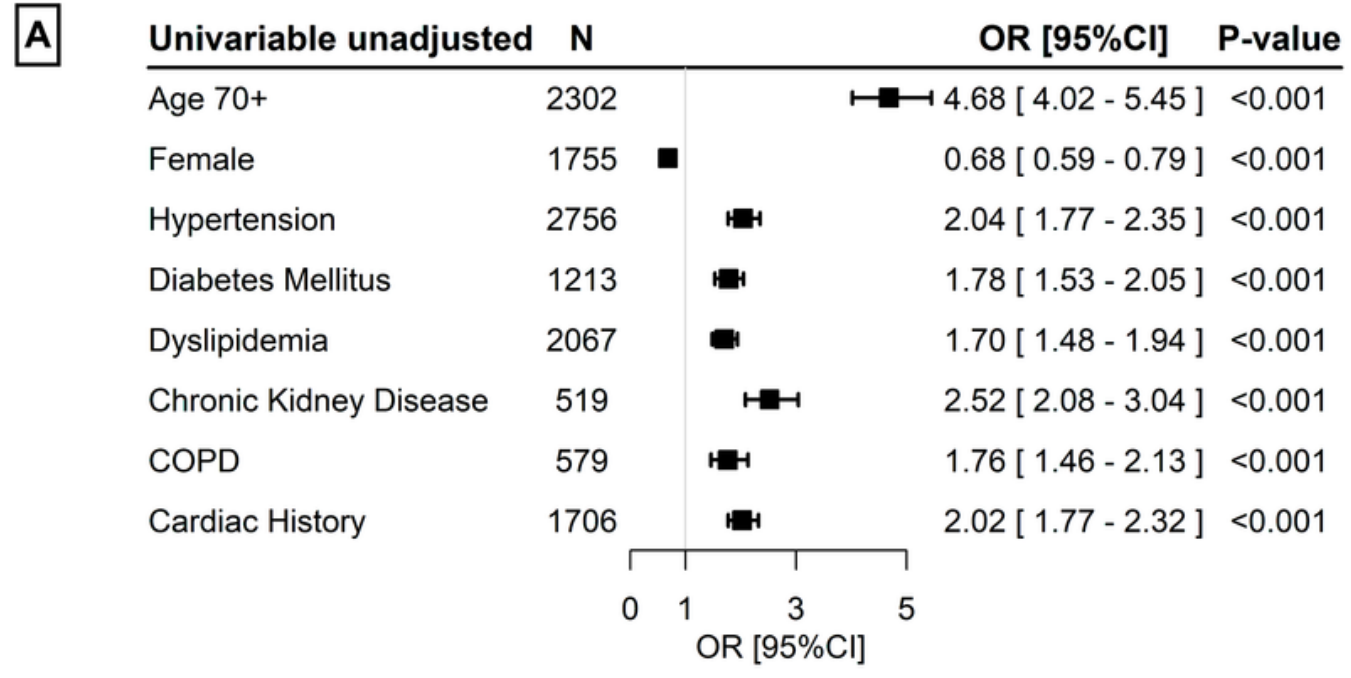

\begin{tabular}{|c|c|c|c|c|}
\hline Age $70+\&$ Sex adjusted & $\mathbf{N}$ & & OR $[95 \% \mathrm{Cl}]$ & P-value \\
\hline Hypertension & 2756 & $=$ & $1.29[1.10-1.51]$ & 0.001 \\
\hline Diabetes Mellitus & 1213 & $\sin$ & $1.55[1.33-1.81]$ & $<0.001$ \\
\hline Dyslipidemia & 2067 & - & $1.15[1.00-1.34]$ & 0.053 \\
\hline Chronic Kidney Disease & 519 & $H$ & $1.74[1.43-2.13]$ & $<0.001$ \\
\hline COPD & 579 & $\boldsymbol{H}$ & $1.41[1.15-1.72]$ & $<0.001$ \\
\hline \multirow[t]{3}{*}{ Cardiac History } & 1706 & - & $1.22[1.05-1.41]$ & 0.010 \\
\hline & Г & 1 & & \\
\hline & 0 & $\left.\begin{array}{lc}1 & 3 \\
\text { OR }[95 \% \mathrm{Cl}\end{array}\right]$ & 5 & \\
\hline
\end{tabular}

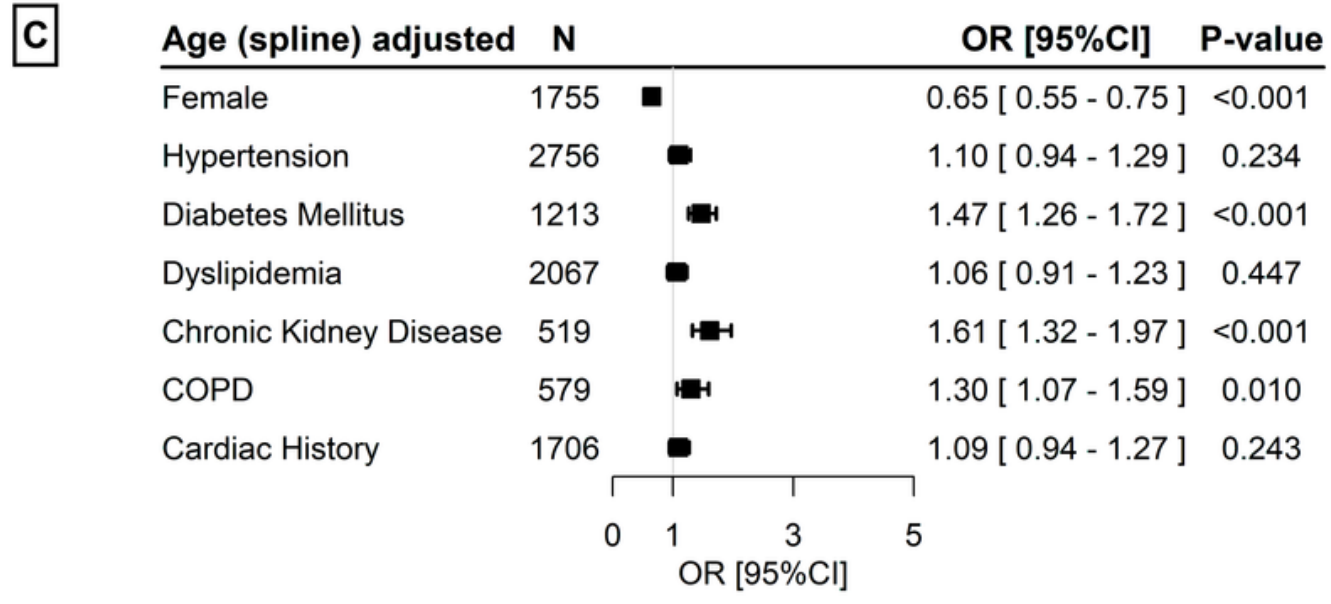

\section{Figure 2}

Associations of age and comorbidities with in-hospital mortality. A) Univariable association of age 70+, sex, and comorbidities with in-hospital mortality; B) Association of all comorbidities after adjustment for age $70+$ and sex; $C$ ) Association of sex and all comorbidities after age spline adjustment. COPD= chronic obstructive pulmonary disease. 


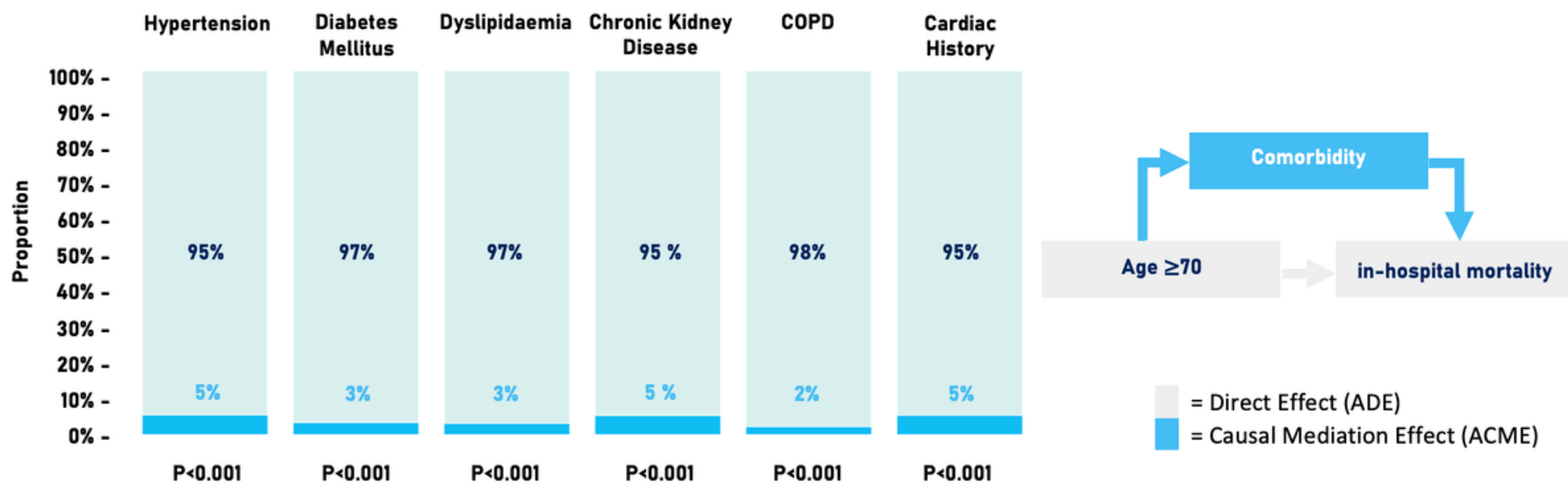

\section{Figure 3}

Mediation analysis with age $\geq 70$ years as independent predictor, the individual comorbidities as mediator and in-hospital mortality as outcome. All comorbidities partly mediated the effect of age on in-hospital mortality (all p-values $<0.001)$. The mediation effect was $5 \%(95 \% \mathrm{Cl} 2-9 \%)$ for hypertension, $3 \%(95 \% \mathrm{Cl}$ $2-4 \%$ ) for diabetes mellitus, $3 \%$ (95\% Cl 0.4-6\%) for dyslipidemia, 5\% (95\% Cl 3-7\%) for chronic kidney disease, $2 \%$ (95\% Cl 1-3\%) for COPD, and $5 \%$ (95\% Cl 2-9\%) for history of cardiac disease. COPD= chronic obstructive pulmonary disease.

\section{Supplementary Files}

This is a list of supplementary files associated with this preprint. Click to download.

- Supplementalmaterial.docx 